\title{
Lesiones de los sargentos alumnos del Ejército de Tierra y factores de riesgo lesional
}

\author{
Valero Capilla FA. ${ }^{1}$, Franco Bonafonte L. ${ }^{2}$, Rubio Pérez FJ. ${ }^{2}$ \\ Sanid. mil. 2014; 70 (4): 263-269; ISSN: 1887-8571
}

\begin{abstract}
RESUMEN
Antecedentes: los cursos de entrenamiento militar requieren un alto nivel de exigencia física y provocan un número importante de lesiones osteomusculares. Es de interés conocer el tipo de lesiones que se observan en estos cursos y los factores de riesgo lesional asociados, para poder implantar posteriormente medidas preventivas adecuadas y correctas gestiones de los recursos que se disponen. Objetivo: presentar el tipo de lesiones observadas durante la fase común del primer curso de formación de los sargentos alumnos del Ejército de Tierra (ET) y analizar la frecuencia lesional y factores de riesgo relacionados. Material y Métodos: estudio descriptivo observacional de corte transversal de 15 semanas de duración, en el que se incluyeron 579 sargentos alumnos (49 mujeres), edad media de 25,9 años (Rango 18-31). Al inicio del curso, se obtuvieron los parámetros antropométricos de los alumnos y se midió su condición física mediante las marcas obtenidas en carrera de 6000,1000 y $50 \mathrm{~m}$, y pruebas de salto vertical y flexo extensiones en suelo. Durante el curso, se registraron las lesiones observadas, sus causas, localización y tiempo de recuperación. Al final del curso, se comparó la frecuencia lesional observada y sus causas entre hombres y mujeres, y en el grupo de hombres, se compararon las marcas obtenidas y los parámetros antropométricos entre los lesionados y no lesionados. Resultados: la edad media del grupo de mujeres vs hombres fue de $27,1(3,18)$ vs $25,7(3,03)$ años $\mathrm{p}<0,01$; la talla $1,63(0,05)$ vs $1,77(0,07) \mathrm{m}, \mathrm{p}<0,0001$; y el peso $57,2(5,04)$ vs 75,2 $(9,14) \mathrm{kg}, \mathrm{p}<0,0001$, respectivamente. Las marcas obtenidas por el grupo de mujeres fueron significativamente menores en todas las pruebas $(p<0,0001)$. Se lesionaron 111 alumnos $(19,1 \%)$, observándose un total de 113 lesiones. Las lesiones mas frecuentes fueron los esguinces y tendinitis que supusieron el $61 \%$ de las lesiones, y la localización más frecuente fue en extremidades inferiores $(87,6 \%)$. Las lesiones se produjeron por sobrecarga en el $54,4 \%$ de los casos frente al $49,6 \%$ por accidente ( $p=N S$ ). Se lesionaron 18 mujeres $(36,7 \%)$ frente a 95 hombres $(17,9 \%), \mathrm{p}<0,05$. En el grupo de hombres, las marcas iniciales de los lesionados frente a no lesionados en extremidades inferiores, fueron las siguientes: en $6 \mathrm{~km}, 28,1(2,40)$ vs $27,0(2,82) \mathrm{min}, \mathrm{p}<0,001$; en $1 \mathrm{~km}, 3,49(0,28)$ vs $3,39(0,24)$ min, $\mathrm{p}<0,001$; en $50 \mathrm{~m}, 7,29(0,41)$ vs $7,18(0,40) \mathrm{seg}, \mathrm{p}<0,05$, respectivamente. No hubo diferencias significativas entre las marcas obtenidas en salto vertical y flexo-extensiones, entre los hombres lesionados y no lesionados, ni tampoco en edad, peso, talla e IMC. Conclusiones: se observa que en torno al $20 \%$ de los alumnos se lesionan durante el curso académico, siendo los esguinces y tendinitis las lesiones más frecuentes. Las lesiones se localizan preferentemente en extremidades inferiores. La mitad de las lesiones se producen por sobrecarga y la otra mitad por accidente. Las mujeres se lesionan más que los hombres. Diferencias antropométricas y una menor condición física pueden determinar su mayor incidencia lesional. En el grupo de hombres, una peor condición física se relaciona con un mayor riesgo lesional, no encontrándose relación de la frecuencia lesional con la edad, peso, talla ni IMC en este grupo.
\end{abstract}

PALABRAS CLAVES: sargentos alumnos, lesiones osteomusculares, factores de riesgo lesional

\section{Injuries of the Ground Forces Student Sergeants and injury risk factors}

SUMMARY: Background: military training courses are very physically demanding and they cause a significant number of musculoskeletal injuries. It is of interest to know the type of injuries observed in these courses and the associated injury risk factors in order to subsequently implant appropriate preventive measures and manage available resources. Objective: to present the type of injuries observed during the common phase of the first training course of the Ground Forces Student Sergeants and to analyze the frequency of injuries and related risk factors. Material and Methods: descriptive, observational, cross-sectional, 15 week duration study, which included 579 student sergeants (49 women), average age of 25.9 years (range 18-31). At the beginning of the course, the anthropometric parameters of students were obtained and their physical condition was measured through the marks obtained in 6000,1000 and $50 \mathrm{~m}$ career, and through vertical jump and flexion/extension tests on the ground. During the course, observed injuries, their causes, location and recovery time were recorded. At the end of the course, the observed frequency of injuries and their causes were compared between men and women, and in the male group, the obtained marks and the anthropometric parameters were compared between the injured and not injured. Results: the average age of the women vs men's group was 27.1 (3.18) vs. 25.7 (3.03) years $p<0,01$; height 1.63 (0.05) vs $1.77(0.07) \mathrm{m}, \mathrm{p}<0.0001$; and weight $57.2(5.04)$ vs $75.2(9.14) \mathrm{kg}, \mathrm{p}<0.0001$, respectively. The marks obtained in the women's group were significantly lower in all tests $(\mathrm{p}<0.0001)$. One hundred and eleven students got injured $(19.1 \%)$, with a total of 113 injuries. The most frequent injuries were sprains and tendinitis which accounted for $61 \%$ of the lesions, and the most frequent location was in

Tcol. Médico. Academia General Básica de Suboficiales del Ejército de Tierra. Talarn (Lérida). España.

Médico. Hospital Universitario de Sant Joan de Reus. Reus (Tarragona). Unidad de Medicina del Deporte. Reus (Tarragona). España.

Dirección para correspondencia: Tcol. Médico Fco. Alfredo Valero Capilla. Academia General Básica de Suboficiales del ET. Ctra. de Talarn s/n 25640 Talarn (Lérida). Email: fvalca2@et.mde.es

Recibido: 23 de enero de 2014

Aceptado: 5 de marzo de 2014 lower limbs (87.6\%). Overload injuries occurred in $54.4 \%$ of cases compared to $49.6 \%$ caused by accident $(\mathrm{p}=\mathrm{NS})$. Eighteen women got injured $(36.7 \%)$ against 95 men $(17.9 \%), p<0.05$. In the male group, the initial marks in the subgroup with lower limbs injuries compared to the not injured, were the following: $6 \mathrm{~km}, 28.1(2.40)$ vs $27.0(2.82) \mathrm{min}, \mathrm{p}<0.001$; in $1 \mathrm{~km}, 3.49(0.28)$ vs $3.39(0.24) \mathrm{min}$, $\mathrm{p}<0.001$; in $50 \mathrm{~m}, 7.29(0.41)$ vs $7.18(0.40) \mathrm{sec}, \mathrm{p}<0.05$, respectively. There were no significant differences neither in the marks 
obtained in vertical jump and flexion/extensions among the injured and not injured, nor in age, weight, height and BMI. Conclusions: it is observed that around $20 \%$ of the students get injured during the academic year, being the sprains and tendinitis the most frequent injuries. The injuries are predominantly located in lower extremities. Half of the injuries are caused by overload and the other half by accident. Women get injured more than men. Anthropometric differences and a lower physical condition might determine the major incidence of their injuries. In the male group, worse physical condition is related to an increased risk of injury, but the relationship between the frequency of injuries and age, weight, height or BMI is not found in this group.

KEY WORDS: sergeants students, musculoskeletal injuries, lesional risk factors.

\section{INTRODUCCIÓN}

Los cursos de entrenamiento militar requieren un alto nivel de exigencia física y provocan un número importante de lesiones osteomusculares ${ }^{1-3}$. En las instalaciones de adiestramiento militar, las lesiones osteomusculares se producen con una frecuencia doble de la de otras instalaciones militares, y constituyen el primer motivo de demanda asistencial durante los cursos de entrenamiento específico de combate ${ }^{4-7}$. La incidencia lesional no solo tiene importancia médica, sino también operativa, ya que conllevan la inactividad física del alumno y perdida de la eficiencia general del curso. En diferentes ejércitos, se ha descrito que cada mes de entrenamiento se lesiona en torno al 10-12\% del personal militar ${ }^{2}$. La incidencia y localización de las lesiones durante los periodos de adiestramiento, dependen en gran parte del tipo de actividad física que se realiza y del nivel de exigencia física requerida ${ }^{2,8-10}$. Factores como el sexo, edad, antropometría, $\mathrm{u}$ otros personales o ambientales, se han descrito también como condicionantes de la incidencia y características de las lesiones observadas durante este tipo de cursos ${ }^{1,4,11-12}$.

El plan actual de formación de los sargentos en el Ejército español, dura 3 cursos académicos y se desarrolla en diferentes academias militares. El primer curso se desarrolla en dos fases, la primera o fase común a todas las especialidades, se realiza en la Academia General Básica de Suboficiales de Talarn, y la segunda fase y el resto de cursos se realiza en otras academias, dependiendo de la especialidad a la que optan los alumnos. En la fase inicial común, los sargentos alumnos deben desarrollar un programa de preparación física general y de entrenamiento básico de combate, que previsiblemente causan frecuentes lesiones osteomusculares, como se ha descrito en otros ejércitos ${ }^{13}$.

Es de interés conocer el tipo y la frecuencia de las lesiones que se producen durante los cursos militares y los factores de riesgo lesional asociados, al objeto de detectar ejercicios y grupos de población de mayor riesgo y poder aplicar planes de prevención concretos $^{14}$. Sin embargo, existen pocos datos publicados que se refieran a los cursos de formación en las academias militares españolas. El objetivo principal de este estudio es el de presentar el tipo, localización y prevalencia de las lesiones observadas durante la fase inicial de formación de los sargentos alumnos del ET, y analizar sus causas y factores de riesgo relacionados.

\section{MATERIAL Y MÉTODOS}

Se realizó un estudio descriptivo observacional de corte transversal de 15 semanas de duración. Se incluyeron 579 alumnos con edad media de 25,9 años, que formaban la totalidad de los alumnos de la XL Promoción de Acceso a la Escala de Subo- ficiales del ET. El curso de formación se realizó del 1 septiembre al 20 de diciembre de 2012, en la Academia General Básica de Suboficiales del ET, de Talarn (Lérida), ubicada en zona montañosa del prepirineo, a una altitud entre 600-700 m.

El programa de actividades físicas que desarrollaron los alumnos consistió en entrenamiento físico general, fundamentalmente de resistencia, sobre todo carrera en terreno con gran desnivel; y entrenamiento específico de campo y simulación de guerra, que consistió en marchas logísticas y topográficas diurnas y nocturnas, despliegues de ataque y defensa y paso de pista de obstáculos (Pista de Combate). Los ejercicios de campo se realizaron portando equipo y armamento. El total de horas de actividad física programada fue de $45 \mathrm{~h}$ de educación física y $145 \mathrm{~h}$ de ejercicios de campo y simulación de guerra.

Al inicio del curso se midió la condición física de los alumnos mediante pruebas de velocidad explosiva $(50 \mathrm{~m})$, resistencia en carrera (1000 y $6000 \mathrm{~m})$, y potencia de tren inferior (salto vertical) y superior (flexo-extensiones de brazos en suelo). El salto vertical se midió como la diferencia entre la altura máxima alcanzada en el salto vertical menos la de bipedestación, permitiendo en el impulso la flexión de piernas. Las flexo-extensiones de brazos se midieron como el número máximo de extensiones realizadas en 2 minutos o hasta el agotamiento. El desarrollo de las pruebas físicas se llevo a cavo por los instructores del departamento de Educación Física de la Academia, los cuales se encargaron asimismo de la toma y registro de marcas. Las pruebas forman parte de la evaluación de los alumnos, por lo que estuvieron altamente motivados en la obtención de marcas. Las pruebas se realizan de acuerdo a la normativa académica del Ejército ${ }^{15}$.

Durante el curso, las lesiones se diagnosticaron siguiendo criterios de diagnóstico clínico, apoyados en su caso por pruebas de imagen, y se anotaron en los correspondientes historiales médicos. En el estudio solo se incluyeron las lesiones que originaron al menos 1 día de baja médica o "limitación de actividades físicas", considerándose leves, moderadas o graves si el periodo de baja fue inferior a 8 días, entre 8 y 28 días o más de 28 días respectivamente, de acuerdo a la categorización de severidad publicada en la guía médica de FIFA ${ }^{16}$. Asimismo, las lesiones se clasificaron según el mecanismo de producción en: lesiones por accidente (traumáticas/agudas) o lesiones de sobrecarga (sobre uso), según se hubiera referido o no accidente traumático inmediatamente previo a la lesión.

Al final del curso, se analizaron los datos obtenidos del registro académico de marcas, de los historiales médicos y del libro de reconocimiento, donde se anota diariamente la situación médica de los alumnos. La edad de los alumnos se obtuvo de una base de datos administrativa que contiene las fechas de nacimiento, y el peso y talla fueron referidos por el alumno, aunque en caso de duda se les tallo y peso. Se ha de señalar que los investigadores 
Tabla 1. Características antropométricas de los sargentos alumnos.

\begin{tabular}{|lcccc|}
\hline & $\begin{array}{c}\text { TODOS } \\
\mathrm{n}=579\end{array}$ & $\begin{array}{c}\text { MUJERES } \\
\mathrm{n}=49\end{array}$ & $\begin{array}{c}\text { HOMBRES } \\
\mathrm{n}=530\end{array}$ & Valor de $\mathrm{p} *$ \\
\hline Edad (años) & $25,9(3,06)$ & $27,1(3,18)$ & $25,7(3,03)$ & $\mathrm{p}<0,01$ \\
Talla $(\mathrm{m})$ & $1,75(0,07)$ & $1,63(0,05)$ & $1,77(0,07)$ & $\mathrm{p}<0,0001$ \\
Peso $(\mathrm{kg})$ & $73,9(9,74)$ & $57,2(5,04)$ & $75,2(9,14)$ & $\mathrm{p}<0,0001$ \\
IMC $\left(\mathrm{kg} / \mathrm{m}^{2}\right)$ & $23,9(2,17)$ & $21,6(1,78)$ & $24,1(2,31)$ & $\mathrm{p}<0,0001$ \\
\hline
\end{tabular}

Los datos se presentan como Medias \pm Desviación Estándar.

* El valor de p se refiere a comparaciones entre Hombres vs Mujeres, p significativa $<0,05$

Tabla 2. Resultados de las pruebas físicas en hombres vs mujeres.

\begin{tabular}{|lccc|}
\hline PRUEBA & $\begin{array}{c}\text { MUJERES } \\
\mathrm{n}=49\end{array}$ & $\begin{array}{c}\text { HOMBRES } \\
\mathrm{n}=530\end{array}$ & Valor de $\mathrm{p}$ \\
\hline $6 \mathrm{~km}(\mathrm{~min})$ & $30,3(4,77)$ & $27,2(2,78)$ & $\mathrm{p}<0,0001$ \\
$1 \mathrm{~km}(\mathrm{~min})$ & $4,06(0,27)$ & $3,41(0,25)$ & $\mathrm{p}<0,0001$ \\
$50 \mathrm{~m}(\mathrm{seg})$ & $8,22(4,06)$ & $7,20(0,40)$ & $\mathrm{p}<0,0001$ \\
Salto vertical (cm) & $41,18(4,62)$ & $52,24(5,82)$ & $\mathrm{p}<0,0001$ \\
Flexo-extensiones (núm) & $20,8(9,36)$ & $36,1(10,4)$ & $\mathrm{p}<0,0001$ \\
\hline
\end{tabular}

Tabla 3. Diagnósticos y localización de las lesiones.

\begin{tabular}{|c|c|c|c|}
\hline & $\begin{array}{c}\text { LESIONES } \\
n=113(\%)\end{array}$ & $\begin{array}{c}\text { DIAS DE } \\
\text { REPOSO } \\
\text { FISICO } \\
n=1340(\%)\end{array}$ & $\begin{array}{c}\text { MEDIA DE } \\
\text { DIAS DE } \\
\text { BAJA (DE) } \\
\#\end{array}$ \\
\hline \multicolumn{4}{|l|}{ Diagnostico* } \\
\hline Tendinitis & $35(30,9)$ & $278(20,7)$ & $7,9(3,52)$ \\
\hline Esguince & $34(30,0)$ & $372(27,8)$ & $10,9(2,51)$ \\
\hline Rotura fibrilar & $7(6,2)$ & $64(4,8)$ & $9,1(3,78)$ \\
\hline Contusión o herida traumática & $12(10,7)$ & $93(6,9)$ & $7,7(5.99)$ \\
\hline Fractura o rotura de tendón & $3(2,7)$ & $265(19,8)$ & $88,3(46,4)$ \\
\hline Algia osteomuscular inespecífica & $18(15,9)$ & $120(9,0)$ & $6,6(2,25)$ \\
\hline Otras lesiones & $4(3,5)$ & $148(11,0)$ & $37,0(50,7)$ \\
\hline \multicolumn{4}{|l|}{ Localizacion* } \\
\hline Cabeza o torax & $2(1,8)$ & $32(2,4)$ & $16,5(2,83)$ \\
\hline Espalda/region dorsolumbar & $6(5,3)$ & $246(18,4)$ & $41,0(58,5)$ \\
\hline Extremidades superiores & $6(5,3)$ & $178(13,3)$ & $29,7(41,4)$ \\
\hline Muslos & $9(8,0)$ & $78(5,8)$ & $8,6(7,76)$ \\
\hline Rodillas & $33(29,2)$ & $284(21,2)$ & $10,5(7,08)$ \\
\hline Piernas & $18(15,9)$ & $153(11,4)$ & $8,5(4,40)$ \\
\hline Tobillos & $32(28,3)$ & $339(25,3)$ & $10,5(9,71)$ \\
\hline Pies & $7(6,2)$ & $30(2,2)$ & $4,29(2,43)$ \\
\hline
\end{tabular}

* Los porcentajes se refieren al total de lesiones.

\# La media global de días de baja de actividades físicas por lesión fue de 11,8 (17,5) días.

no conocieron las marcas obtenidas en las pruebas físicas, hasta el final del curso que se analizaron los datos.

Para el tratamiento de datos se ha empleado el paquete informático GraphPad InStat tm V2.05a, y el paquete de funciones estadísticas Microsoft Excel 97-2000. Se ha utilizado la $t$ de Student para comparación de medias con distribución normal, y el test exacto de Fisher para proporciones. Al valorar la diferencia de medias o de proporciones se ha considerado como estadísticamente significativo un valor de $\mathrm{p}<0,05$.

\section{RESULTADOS}

\section{Características antropométricas y pruebas físicas iniciales}

Las características antropométricas de los sargentos alumnos, se presentan en la Tabla 1. La media de edad fue de 25,9 (3,06 años), rango 18-31, y el IMC de 23,9 (2,17), rango 18-29. La edad media fue algo superior en mujeres que en hombres: $27,1(3,18)$ vs $25,7(3,03)$ años, respectivamente, $\mathrm{p}=$ 0,0038. La talla, peso e IMC fueron superiores en hombres con diferencias altamente significativas.

Los resultados de las pruebas físicas en hombres y mujeres se presentan en la Tabla 2. En todas las pruebas las mujeres obtuvieron menores marcas con una alta significación estadística $(\mathrm{p}<0001)$.

\section{Prevalencia y características de las lesiones}

Para el diagnóstico de las lesiones se solicitaron las siguientes pruebas complementarias: 10 Radiografías simples, 10 Ecografías, 10 Resonancias y 3 Tomografías.

Se observaron un total 113 lesiones. El 19,1\% de los alumnos presentó alguna lesión. La incidencia fue de 5,57 lesiones por 100 individuos por mes. El tipo de lesiones sufridas se expone en la Tabla 3. Dos individuos sufrieron dos lesiones, el resto presentó una lesión. Los diagnósticos más frecuentes fueron esguinces y tendinitis, que juntos supusieron un total de 69 lesiones $(61,0 \%)$. Hubo 2 fracturas, una vertebral, en una alumna y una de metacarpiano en un alumno. En un caso se diagnosticó inicialmente un esguince de hombro, pero posteriormente se comprobó una rotura tendinosa, por la que el alumno fue intervenido meses después. En el apartado de "Otras lesiones" de la Tabla 3, se incluyeron: 1 periostitis tibial, 2 condropatías rotulianas, y 1 hernia discal no traumática.

La localización más frecuente de las lesiones fue en extremidades inferiores (EEII), donde se registraron 99 lesiones (87,6\%), Tabla 3. Las zonas más lesionadas fueron las rodillas y tobillos en las que se localizaron un total de 65 lesiones $(58,5 \%)$.

Las lesiones supusieron 1340 días de baja de actividades físicas, lo que supuso una media de 11,8 $(17,5)$ días de baja por 
Tabla 4. Causas de las lesiones.

\begin{tabular}{|lcccc|}
\hline & $\begin{array}{c}\text { TODOS } \\
\text { ALUMNOS n } \\
=579\end{array}$ & $\begin{array}{c}\text { MUJERES } \\
\mathrm{n}=49\end{array}$ & $\begin{array}{c}\text { HOMBRES } \\
\mathrm{n}=530\end{array}$ & Valor de $\mathrm{p} *$ \\
\hline Todas las lesiones (\% alumnos lesionados) & $113(19,1)$ & $18(36,7)$ & $95(17,9)$ & $\mathrm{p}<0,05$ \\
1. Sobrecarga (\$) & $57(50,4)$ & $6(33,3)$ & $51(53,6)$ & $\mathrm{p}=\mathrm{NS}$ \\
2. Accidente (\$) & $56(49,6)$ & $12(66,6)$ & $44(46,3)$ & $\mathrm{p}<0,01$ \\
- Educación física (\#) & $11(19,6)$ & $3(25,0)$ & $8(18,1)$ & $\mathrm{p}=\mathrm{NS}$ \\
- Instrucción de campo (\#) & $36(64,2)$ & $3(25,0)$ & $33(75,0)$ & $\mathrm{p}=\mathrm{NS}$ \\
- Pista de combate (\#) & $9(16,0)$ & $6(50,0)$ & $3(6,8)$ & $\mathrm{p}<0,0001$ \\
\hline
\end{tabular}

$\$$ Porcentajes sobre el total de lesiones de cada grupo analizado (de cada columna).

\# Porcentajes sobre el total de lesiones por accidente en cada grupo analizado.

* El valor de p se refiere a comparaciones entre hombres vs mujeres.

Tabla 5. Comparaciones entre características de hombres lesionados vs no lesionados en extremidades inferiores.

\begin{tabular}{|lccc|}
\hline & $\begin{array}{c}\text { HOMBRES LESIONADOS } \\
\mathrm{n}=83\end{array}$ & $\begin{array}{c}\text { HOMBRES NO } \\
\text { LESIONADOS } \\
\mathrm{n}=432\end{array}$ & Valor de $\mathrm{p}$ \\
\hline Edad (años) & & $25,9(4,42)$ & $\mathrm{p}=\mathrm{NS}$ \\
Peso (kg) & $25,8(3,14)$ & $75,4(9,05)$ & $\mathrm{p}=\mathrm{NS}$ \\
Talla (m) & $74,3(9,69)$ & $1,77(0,06)$ & $\mathrm{p}=\mathrm{NS}$ \\
IMC (kg/m $\left.{ }^{2}\right)$ & $1,76(0,07)$ & $\mathrm{p}=\mathrm{NS}$ \\
Marca inicial en $6 \mathrm{~km}(\mathrm{~min})$ & $23,9(2,12)$ & $\mathrm{p}<0,001$ \\
Marca inicial en $1 \mathrm{~km}(\mathrm{~min})$ & $28,1(2,40)$ & $\mathrm{p}<0,001$ \\
Marca inicial en $50 \mathrm{~m}(\mathrm{seg})$ & $3,49(0,28)$ & $\mathrm{p}<0,05$ \\
Salto vertical $(\mathrm{cm})$ & $7,29(0,41)$ & $3,39(0,24)$ & $\mathrm{p}=\mathrm{NS}$ \\
Flexo-extensiones $\left(\mathrm{n}^{\mathrm{o}}\right)$ & $51,8(5,93)$ & $7,18(0,40)$ & $\mathrm{p}=\mathrm{NS}$ \\
\hline
\end{tabular}

lesión, rango 1-120 días (Tabla 3). Las lesiones que mas días de baja provocaron fueron, la fractura vertebral, la hernia discal lumbar y la rotura tendinosa de hombro, que juntas supusieron 343 días de baja $(25,7 \%)$. En cuanto a la severidad de las lesiones, $66(58,4 \%)$ fueron leves, con duración entre 1 y 7 días, 42 $(31,5 \%)$ moderadas y $5(4,4 \%)$ fueron graves, con una duración superior a 28 días. No hubo diferencia entre sexos en la distribución de la severidad de las lesiones.

\section{Causas y factores de riesgo}

El tipo de actividades físicas que realizaban los alumnos en el momento de lesionarse se presenta en la Tabla 4. Hubo 57 lesiones por sobrecarga/sobre uso, que supusieron la mitad de las lesiones $(50,4 \%)$ frente a $56(49,6 \%)$ por accidente/traumáticas $(p=N S)$. Las lesiones por accidente se produjeron principalmente durante las actividades de instrucción específica de campo y simulación de guerra. Durante estas actividades se produjeron 45 lesiones por accidente (80,3\% de los accidentes), el resto de lesiones por accidente se produjeron durante la educación física. La fractura vertebral y la rotura tendinosa de hombro se produjeron en el paso de la Pista de Combate.

Las mujeres se lesionaron proporcionalmente más que los hombres: $18(36,7 \%)$ vs $95(17,9 \%)$, respectivamente, $\mathrm{p}=0,039$
(Tabla 4), y sufrieron mas lesiones por accidente $12(24,4 \%)$ en mujeres vs $44(8,3 \%)$ en hombres, $p=0,0012$; Mientras que las lesiones por sobrecarga fueron similares en ambos sexos $6(12,2 \%)$ en mujeres frente a $51(9,6 \%)$ en hombres $\mathrm{p}=\mathrm{NS}$. El paso de la Pista de Combate causó 6 accidentes en mujeres (12,2\%) frente a $3(0,5 \%)$ en los hombres, $p<0,0001$. De hecho, la mitad de los accidentes sufridos por las mujeres, se produjo en la Pista de Combate.

En el grupo de hombres, dado que la mayoría de lesiones se produjeron en extremidades inferiores, se analizó la relación de varios parámetros con la frecuencia lesional observada en esa localización. Solo se analizó el grupo de hombres porque en el de mujeres la muestra de lesiones fue escasa. Así, en los hombres, las marcas de tiempo de carrera en 50, 1000 y $6000 \mathrm{~m}$, registradas al inicio del curso, fueron peores significativamente en los que se lesionarían posteriormente (Tabla 5). Las marcas medias de las pruebas de salto vertical y flexo-extensiones también fueron peores en los lesionados, pero las diferencias no alcanzaron significación estadística (Tabla 5). Al analizar por separado la relación de la condición física con las lesiones por sobrecarga $(n=47)$ o por accidente $(n=47)$, se observó que se mantuvo la relación con las marcas en 6 y $1 \mathrm{~km}$. Así en los hombres lesionados por sobrecarga frente a no lesionados, las marcas medias observadas en $6 \mathrm{~km}$ fueron: $28,3(2,85)$ vs 27,08 $(2,82) \mathrm{min}$, respectivamente, $\mathrm{p}=0,0029$ (Grafico 1$)$, y en $1 \mathrm{~km}: 3,53(0,33)$ vs $3,39(0,24) \mathrm{min}$, 


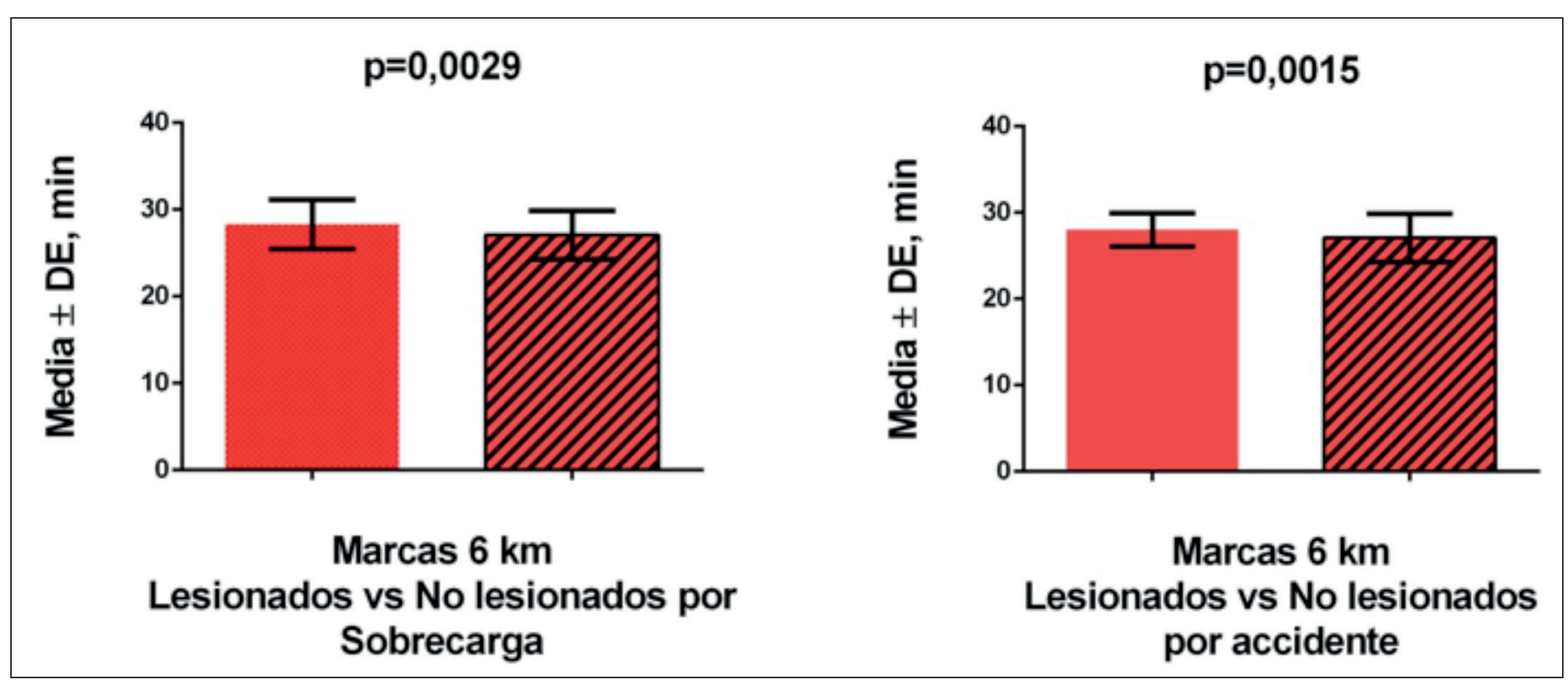

Figura 1. Diferencias de las marcas en $6 \mathrm{~km}$ de Lesionados vs No Lesionados. Las barras lisas corresponden a medias de las marcas obtenidas por los alumnos lesionados y las barras rayadas por los no lesionados.

respectivamente, $\mathrm{p}=0,0047$. Mientras que en los lesionados por accidente frente a no lesionados, las marcas fueron en $6 \mathrm{~km}: 28,0$ $(1,96)$ vs $27,08(2,82) \mathrm{min}$, respectivamente, $\mathrm{p}=0,0015$ (Grafico $1)$, y en $1 \mathrm{~km}: 3,47(0,25)$ vs $3,39(0,24) \mathrm{min}$, respectivamente, $\mathrm{p}=0,0362$. La edad, peso, talla e IMC no mostraron diferencia entre los hombres lesionados y no lesionados (Tabla 5).

\section{DISCUSIÓN}

\section{Características de las lesiones y prevalencia lesional}

El tipo de lesiones más frecuentes observadas en este estudio fueron los esguinces y tendinitis, que representaron el $61 \%$ de todas las lesiones, y la localización más frecuente fue en extremidades inferiores (EEII), donde se localizaron el 87\% de las lesiones, preferentemente en tobillos y rodillas. Estudios de otros ejércitos en cursos similares, encuentran el mismo tipo de lesiones y localización preferente. Almeida y cols., siguieron una población de 1.296 marines americanos, durante un curso de entrenamiento básico de combate de 12 semanas de duración, y observaron que el $82 \%$ de las lesiones se localizaron en EEII, principalmente en tobillos primero y después en rodillas ${ }^{8}$. Asimismo, Jordaan y cols., en el ejército sudafricano siguieron a casi 1300 reclutas durante un curso de 9 semanas, y observaron que más del 80\% de las lesiones se localizaron en tobillos, rodillas y piernas, siendo las lesiones por sobreuso las más frecuentes ${ }^{17}$. Springer y cols., en una monografía reciente, sobre "Lesiones osteomusculares en mujeres militares", describen también que en torno al $85 \%$ de las lesiones se producen en EEII, siendo los principales diagnósticos, las lesiones de sobrecarga en tobillos y rodillas ${ }^{12}$. Finalmente, otros estudios con muestras de poblaciones muy extensas han descrito datos similares tanto en hombres como en mujeres ${ }^{3,18}$.

En nuestro estudio, la fase de adiestramiento duró 15 semanas y encontramos que cerca del $20 \%$ de los alumnos se lesionaron durante el curso, con una incidencia lesional en torno a $6 \%$ de lesionados por mes. Esta incidencia lesional es menor a la encontrada en algunos estudios. En el ejército danés se observó que el $29 \%$ de los militares se lesionaron durante un periodo de entrenamiento de 20 semanas $^{5}$, y en una revisión en el ejército americano se describió una incidencia lesional media de entre el 6 y $12 \%$ de lesionados por mes, en cursos de preparación militar básica, y este porcentaje aumentó hasta el 30\% por mes, en cursos de entrenamiento avanzado de Fuerzas Especiales ${ }^{1}$. Estos y otros autores relacionan la alta incidencia lesional con el alto nivel de exigencia física de los cursos ${ }^{1,3}$. En nuestro estudio aunque el número de alumnos lesionados fue importante, sin embargo la incidencia lesional, comparada con estos estudios no fue tan alta. Quizá, diferencias en el nivel de exigencia física requerida o en el tipo de ejercicios físicos aplicados, explican la menor incidencia lesional observada en nuestro estudio. Por otra parte, en nuestro estudio solo se incluyeron las lesiones que originaron baja médica, mientras que en otros estudios se incluyeron todas las consultas médicas, independientemente de si originaron o no baja médica.

\section{Causas y factores de riesgo lesional}

La mitad de las lesiones en este estudio, se produjeron por sobrecarga y la otra mitad por accidente. Las lesiones observadas, se produjeron sobre todo durante las actividades de campo y simulación de guerra. Como se ha descrito en otros estudios el tipo de actividades físicas realizadas y el tiempo de dedicación a ellas, condiciona la frecuencia y el tipo de lesiones,2,-10. Jordaan y cols., observaron que la mayor incidencia de lesiones apareció en las semanas en las que se dedicaba más tiempo a marchas de campo ${ }^{17}$, y Almeida y cols., describieron que la incidencia lesional semanal se correlacionó positivamente con la intensidad y volumen del entrenamiento de la semana ${ }^{8}$. Asimismo, Springer y cols., describen que las actividades de campo son la principal 
causa de lesiones en mujeres militares ${ }^{12}$. En nuestro estudio se dedicó el triple de tiempo a actividades de campo frente a preparación física general, y los ejercicios de campo se realizaron portando mochila y/o armamento. Además, las marchas y despliegues fueron con frecuencia nocturnos por terreno muy desigual. Todo ello, seguramente determinó que las lesiones se produjeran sobre todo durante las actividades de campo.

\section{Factores lesionales en las mujeres}

En nuestro estudio, las mujeres se lesionaron más que los hombres, acosta de sufrir más accidentes. El paso de la Pista de Combate fue la causa más importante de accidente en ellas, de hecho fue la causa de la mitad de los accidentes que sufrieron. La mayor prevalencia lesional en las mujeres coincide con lo descrito en otros estudios. Snedecor y cols., con una muestra poblacional de más de 13000 militares, de los que 5.250 eran mujeres, describen una prevalencia lesional de un $30 \%$ en las mujeres frente a un $16 \%$ en los hombres ${ }^{19}$. Asimismo Springer y cols., en el ejército americano, citan una prevalencia lesional del $52 \%$ en mujeres frente al $26 \%$ en hombres, durante periodos de adiestramiento básico, y del $30 \%$ en mujeres frente al $24 \%$ en hombres, en entrenamientos avanzados ${ }^{12}$. Otros estudios obtienen resultados similares ${ }^{2,20,21}$. Diferencias antropométricas o biomecánicas y la menor condición física de las mujeres, parecen determinar su mayor incidencia lesional ${ }^{12}$. Blacker y cols., en un estudio británico, determinaron el esfuerzo cardiovascular de militares de ambos sexos, mediante la aplicación individual de un pulsómetro y un acelerómetro, y describieron que las mismas actividades físicas, produjeron en las mujeres un mayor esfuerzo cardiovascular, y sugieren que ese mayor esfuerzo determinaría mayor fatiga y por tanto, mayor riesgo lesional ${ }^{22}$. Finalmente, Bell y cols., encuentran doble incidencia lesional en mujeres, y en un análisis multivariante en el que incluyeron además del sexo, características antropométricas y condición física aérobica inicial, observaron que esta última mantuvo una relación independiente con el riesgo lesional ${ }^{23}$.

En nuestro estudio, las mujeres obtuvieron marcas peores en todas las pruebas físicas, y en comunicaciones anteriores, hemos descrito que las alumnas presentan un menor consumo de $\mathrm{O}_{2}$, al inicio y a lo largo del curso, calculado mediante test de Cooper $^{24,25}$. Así pues, la menor condición física de las mujeres pudo determinar el que se lesionaran en mayor proporción. Se ha de señalar también que ambos sexos han de pasar los mismos obstáculos de la Pista de Combate, por lo que la menor talla y fortaleza de las mujeres pudo condicionar asimismo el que sufrieran más accidentes en ese ejercicio. De hecho, las mujeres presentaron una talla media $14 \mathrm{~cm}$ menor y un IMC también significativamente más bajo. Finalmente en nuestro estudio la edad de las mujeres fue algo mayor que la de los hombres, lo que también pudo influir en su mayor frecuencia lesional ${ }^{11}$. Al contrario de estos resultados, Almeida y cols., aunque registraron una mayor proporción de mujeres lesionadas, sin embargo al interrogar por las lesiones no declaradas e incluirlas también en el análisis, encontraron que ambos sexos se habían lesionado en la misma proporción, pero las mujeres habían acudido más a consulta médica, por lo que tenía registradas más lesiones ${ }^{26}$.

\section{Factores lesionales en los hombres}

En el grupo de hombres, observamos que la frecuencia de lesiones no se relacionó con la edad ni las características antropométricas. Kaufman y cols., en una revisión epidemiológica de varias unidades del ejército americano encontraron relación de la edad con la incidencia lesional, aunque, según ellos mismos reseñan, los datos fueron contradictorios ${ }^{1}$. En otros estudios se ha descrito relación entre algunos tipos de lesiones y el peso, talla o edad ${ }^{11}$. Nuestra población tenía un estrecho rango de edad y características antropométricas, hecho que probablemente impidió encontrar cualquier relación de estos parámetros con la prevalencia lesional.

Por otra parte, en los hombres, la frecuencia de lesiones se relacionó a la condición física inicial. Así, los alumnos que mostraron peores marcas en las pruebas de carrera de $50 \mathrm{~m}, 1 \mathrm{~km}$ y $6 \mathrm{~km}$ sufrieron mas lesiones en EEII. En otros estudios ya se ha descrito esta relación ${ }^{11,17,23,27}$. Rosendal y cols., en una población militar danesa, a la que determinó la condición fisca inicial mediante un test de Cooper, encontró que los reclutas con menor forma física sufrieron más lesiones por sobrecarga ${ }^{5}$. Asimismo, Neely, en una revisión de población militar británica, describe esta misma relación con lesiones en EEII ${ }^{11}$. Finalmente, Knapik y cols., determinaron el consumo de $\mathrm{O}_{2}$ y condición física en militares americanos, mediante carrera en cinta, prueba de 2 millas, flexo-extensiones de brazos y flexión de abdominales, y observaron que la $\mathrm{VO}_{2}$ máxima, tiempo de carrera y el número de flexo-extensiones se asociaron al riesgo lesional, tanto en hombres como en mujeres ${ }^{27}$. Nosotros solo encontramos relación con los tiempos de carrera, y aunque las marcas en salto vertical y en flexo-extensiones fueron peores en los lesionados, la diferencia no fue significativa.

Un hallazgo interesante de nuestro estudio fue que la menor condición física mostró también relación con las lesiones producidas por accidente, es decir, los sargentos alumnos peor preparados físicamente al inicio del curso, sufrieron no solo mas lesiones por sobrecarga sino también más lesiones por accidente. En otros estudios no se describe esta relación. En el estudio de Rosendal y cols., la mayoría de las lesiones fueron por sobre uso, desconocemos si se analizó la asociación con las lesiones por accidente ${ }^{5}$. Knapik y cols., encontraron una proporción de lesiones por traumatismo en torno al $15 \%$ y no se relacionaron con la condición física ${ }^{27}$. En nuestro estudio casi el 50\% de las lesiones fueron por accidente. Este porcentaje es superior al de otros estudios, quizá diferencias en el programa de ejercicios de adiestramiento aplicado o diferencias en el criterio de clasificación de las lesiones, puedan explicar esa discrepancia. En nuestro estudio se ha encontrado relación de la condición física de explosividad y resistencia de carrera con las lesiones de EEII. Es probable que una peor condición física comporte mayor fatiga y pueda determinar más lesiones, tanto por sobrecarga como por traumatismo.

Finalmente, al contrario de los estudios referenciados con población militar, en un estudio con población civil, se encontró una relación positiva de la forma física inicial y la frecuencia lesional observada posteriormente ${ }^{9}$, es decir, los sujetos mejor preparados presentaron más lesiones. En este estudio los individuos no realizaron el mismo entrenamiento y los autores sugie- 


\section{Lesiones de los sargentos alumnos del Ejército de Tierra y factores de riesgo lesional}

ren que, quizá los individuos con mejor forma física realizaron un entrenamiento más intenso, lo que les pudo provocar más lesiones.

Se ha de señalar que la identificación de factores de riesgo lesional en los periodos de adiestramiento militar, permite aplicar planes de prevención general y modificaciones concretas en la forma e intensidad de los programas de entrenamiento. Diferentes estudios han mostrado que la aplicación de medidas preventivas, disminuye el número de lesiones y el gasto asistencial, mejorando la eficiencia académica ${ }^{2,4,13,14,28}$. Nuestro estudio fue de corte transversal por lo que no se pueden establecer relaciones causales, pero puede ser el inicio de otros estudios observacionales más amplios para poder establecerlas.

\section{CONCLUSIONES}

En la fase inicial de formación de los sargentos del ET, se lesionan en torno al 20\%. Las lesiones más frecuentes son los esguinces y tendinitis, que se localizan sobre todo en rodillas y tobillos. La mitad de las lesiones se producen por sobrecarga y la otra mitad por accidente.

Las mujeres se lesionan más, debido a que sufren más accidentes, sobre todo al pasar la Pista de Combate. Diferencias antropométricas y una menor condición física pueden determinar su mayor frecuencia lesional. En el grupo de hombres, la peor condición física inicial se relaciona a un mayor riesgo de sufrir lesiones por sobrecarga y por accidente, no encontrándose relación de la frecuencia lesional con la edad, peso, talla ni el IMC.

\section{AGRADECIMIENTOS}

A los instructores del departamento de Educación Física de la Academia y en especial al capitán CGA Rafael Ángel Rivero del Castillo, jefe del departamento, por su inestimable y desinteresada colaboración en la realización y toma de marcas de las pruebas físicas. Asimismo nuestro agradecimiento al Tte. Enfermero Esteban Pascau Romero, por su valiosa ayuda en la asistencia diaria de los sargentos alumnos.

\section{BIBLIOGRAFÍA}

1. Kaufman HR, Brodine S Shaffer. Military training-related injuries: surveillance, research, and prevention. Am J Prev Med 2000 Apr, 18 (3 suppl): 53-63

2. Jones BH, Knapik JJ. Physical training and exercise-related injuries. Surveillance, research and injury prevention in military populations. Sports Med. 1999 Feb; 27(2):111-25.

3. Gordon NF, Hugo EP, Cilliers JF. The South African Defence Force physical training programme. Part III. Exertion-related injuries sustained at an SADF basic training centre. S Afr Med J. 1986, Apr 12:69 (8):491-4.

4. US Department of the Army. Prevention and Control of Musculoskeletal Injuries Associated with Physical Training. Washington, DC: DA; 2006. Technical Bulletin Medical 592 (TB MED 592).

5. Rosendal L, Henning L, Jensen A, Kjær M. Incidence of injury and physical performance adaptations during Military Training. Clin J Sport Med 2003; 13:157-163.
6. Shaffer RA, Brodine SK, Ito SI, Le AT. Epidemiology of illness and injury among U.S. Navy and Marine Corps female training populations. Mil Med. 1999 Jan; 164(1):17-21.

7. Lauder TD, Baker SP, Smith GS, Lincoln AE. Sports and physical training injury hospitalizations in the army. Am J Prev Med. 2000 Apr;18(3 Suppl):118-28.

8. Almeida SA, Williams KM, Shaffer RA, Brodine SK. Epidemiological patterns of musculoskeletal injuries and physical training. Med Sci Sports Exerc. 1999 Aug;31(8):1176-82.

9. Hootman JM, Jennifer M, Macera CA, Ainsworth B, et al. Association among Physical Activity Level, Cardiorespiratory Fitness, and Risk of Musculoskeletal Injury. Am. J. Epidemiol. (2001) 154 (3): 251-258.

10. Theron N, Schwellnus N, Derman W, Dvorak Jl. Illness and Injuries in Elite Football Players-A Prospective Cohort Study During the FIFA Confederations Cup 2009. Clinical Journal of Sport Medicine 23(5):379-383.

11. Neely FG. Intrinsic risk factors for exercise-related lower limb injuries. Sports Med 1998 Oct; 26(4):253-63.

12. Springer, Barbara A; Ross, Amy E. Borden Institute, Walter Reed Army Medical Center, Paul J. Dougherty. Musculoskeletal injuries in military women. US Army Medical Department Center \& School 2011. Borden Institute Monograph Series.

13. Smith GS, Dannenberg AL, Amoroso PJ. Hospitalization due to injuries in the military. Evaluation of current data and recommendations on their use for injury prevention. Am J Prev Med. 2000 Apr; 18(3 Suppl):41-53.

14. Bullock SH, Jones BH, Gilchrist J, Marshall SW. Prevention of physical training-related injuries recommendations for the military and other active populations based on expedited systematic reviews. Am J Prev Med. 2010 Jan; 38(1 Suppl):S156-81.

15. Orden $\mathrm{DEF} / 1078 / 2012$, de 21 de mayo, por la que se aprueban las pruebas físicas y marcas a alcanzar en los procesos de selección para el ingreso en los centros docentes militares de formación y para la superación de los planes de estudios de la enseñanza de formación.

16. F-MARC Football Medicine Manual. FIFA Medical Assessment and Research Centre (F-MARC). Federation International de Football Association. FIFA Medical Office. 2nd Edition 2009

17. Jordaan G, Schwellnus MP. The incidence of overuse injuries in military recruits during basic military training. Mil Med. 1994 Jun; 159(6):421-6.

18. Hauret KG, Jones BH, Bullock SH, Canham-Chervak M, Canada S. Musculoskeletal. Injuries Description of an Under-Recognized Injury Problem Among Military Personnel. Am J Prev Med 2010;38(1S):S61-S70).

19. Snedecor MR, Boudreau CF, Ellis BE, Schulman J, Hite M, Chambers B. U.S. Air force recruit injury and health study. Am J Prev Med. 2000 Apr; 18(3 Suppl):129-40.

20. Piantanida NA, Knapik JJ, Brannen S, O’Connor F. Injuries during Marine Corps officer basic training. Mil Med. 2000 Jul; 165(7):515-20.

21. Gemmell IM. Injuries among female army recruits: a conflict of legislation. J R Soc Med. 2002 Jan; 95(1):23-7.

22. Blacker SD, Wilkinson DM, Rayson MP. Gender differences in the physical demands of British Army recruit training. Mil Med. 2009 Aug; 174(8):811-6.

23. Bell NS, Mangione TW, Hemenway D, Amoroso PJ, Jones BH. High injury rates among female army trainees: a function of gender?. Am J Prev Med. 2000 Apr;18(3 Suppl):141-6.

24. Valero FA, Franco L, Rubio FJ. Condición Física aeróbica de los Sargentos alumnos del ET. Comunicación oral, Archivos de Medicina del Deporte 2012; Vol XXIX núm 151:909.

25. Valero FA, Franco L, Rubio FJ. Evolución de la condición física de resistencia de los Sargentos alumnos del Ejército de Tierra. Comunicación oral, Archivos de Medicina del Deporte 2012 sep; Vol XXIX núm 151:881.

26. Almeida SA, Trone DW, Leone DM, Shaffer RA, Patheal SL, Long K. Gender differences in musculoskeletal injury rates: a function of symptom reporting? Med Sci Sports Exerc. 1999 Dec; 31(12):1807-12.

27. Knapik JJ, Sharp MA, Canham-Chervak M, Hauret K, Patton JF, Jones $\mathrm{BH}$. Risk factors for training-related injuries among men and women in basic combat training. Med Sci Sports Exerc. 2001 Jun; 33(6):946-54.

28. Rudzki SJ, Cunningham MJ. The effect of a modified physical training program in reducing injury and medical discharge rates in Australian Army recruits. Mil Med. 1999 Sep; 164(9):648-52. 